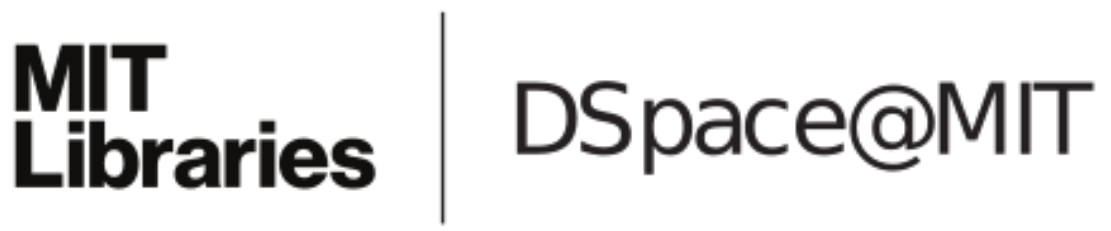

\author{
MIT Open Access Articles
}

\section{In Situ Observation and Mathematical Modeling of Lithium Distribution within Graphite}

The MIT Faculty has made this article openly available. Please share how this access benefits you. Your story matters.

As Published: 10.1149/2.0061711JES

Publisher: The Electrochemical Society

Persistent URL: https://hdl.handle.net/1721.1/134524

Version: Final published version: final published article, as it appeared in a journal, conference proceedings, or other formally published context

Terms of use: Creative Commons Attribution-NonCommercial-NoDerivs License 


\title{
In Situ Observation and Mathematical Modeling of Lithium Distribution within Graphite
}

\author{
Karen E. Thomas-Alyea, ${ }^{\mathrm{a}, *, \mathrm{z}}$ Changhoon Jung, ${ }^{\mathrm{b}}$ Raymond B. Smith, ${ }^{\mathrm{c}}$ and Martin Z. Bazant ${ }^{\mathrm{c}}$ \\ ${ }^{a}$ Samsung Research America, Burlington, Massachusetts 01803, USA \\ ${ }^{b}$ Samsung Advanced Institute of Technology, Yeongtong-gu, Suwon-si, Gyeonggi-do, Korea \\ ${ }^{c}$ Department of Chemical Engineering, Massachusetts Institute of Technology, Cambridge, Massachusetts 02139, USA
}

\begin{abstract}
Lithium forms ordered stages when it reacts with graphite. These stages have distinct colors; therefore, optical microscopy gives direct information about the lithium concentration in the graphite. Here we present in situ optical images during charging and discharging of a graphite electrode. Stages are observed to coexist with each other even after extended rest. There is considerable spatial nonuniformity on the microscale. To predict this concentration distribution, we employ a model which combines porouselectrode theory and Cahn-Hilliard phase-field theory to describe the flux of lithium within the graphite. The model closely matches the experimental voltage and concentration distribution. The spatial nonuniformity can be approximated with a relatively simple model of distributed resistances. Finally, we discuss the implications of using the phase-field model instead of a solid-solution model for prediction of lithium plating. The two models give similar predictions of cell voltage and risk of lithium plating under many operating conditions, with the main difference being the relaxation of concentration gradients within particles during rest. The distributed-resistance model shows a higher risk of lithium plating because well-connected particles are overworked as their more-resistive neighbors require a higher driving force for passage of current.

(c) The Author(s) 2017. Published by ECS. This is an open access article distributed under the terms of the Creative Commons Attribution Non-Commercial No Derivatives 4.0 License (CC BY-NC-ND, http://creativecommons.org/licenses/by-nc-nd/4.0/), which permits non-commercial reuse, distribution, and reproduction in any medium, provided the original work is not changed in any way and is properly cited. For permission for commercial reuse, please email: oa@electrochem.org. [DOI: 10.1149/2.0061711jes] All rights reserved.

(cc) BY-NC-ND
\end{abstract}

Manuscript submitted December 22, 2016; revised manuscript received March 3, 2017. Published March 18, 2017. This paper is part of the JES Focus Issue on Mathematical Modeling of Electrochemical Systems at Multiple Scales in Honor of John Newman.

Graphite, which is used in negative electrodes in most commercial lithium-ion batteries, is known to form ordered stages upon intercalation of lithium. ${ }^{1}$ Intercalation of lithium into the graphite structure proceeds as a stage-transition reaction; for example, at an average composition of 0.8 , the graphite consists of regions of stage 1 and stage 2 in equilibrium with each other. ${ }^{2,3}$ The color and stages of graphite as a function of lithium concentration $\mathrm{x}$ in $\mathrm{Li}_{\mathrm{x}} \mathrm{C}_{6}$ are shown in Table I.

Graphite has a planar crystal structure. Lithium diffusion is believed to be facile parallel to the planes, but diffusion perpendicular to the planes is believed to occur only at crystal defects. ${ }^{4}$

Accurate prediction of the lithium concentration within the electrode is important for understanding failure mechanisms such as lithium plating and stress-induced cracking. We present in situ optical images of the graphite obtained during charging and discharging. Then we apply a model which incorporates stage transitions within particles using Cahn-Hilliard phase-field theory, along with modeling current distribution using porous electrode theory. Most battery simulations have used the mathematics of solid solution to simulate the behavior of the graphite electrode..$^{5-7}$ We assess the conditions under which a phase-transition model would give different predictions from a solid-solution model, particularly with respect to prediction of lithium plating. Finally, the in situ observations motivate us to consider a simplified model to account for spatial nonuniformity within a porous electrode.

\section{Experimental Methods and Observations}

Graphite powder was mixed with binder, slurry coated onto copper foil, calendared to a thickness of $97 \mu \mathrm{m}$, and punched into discs. As shown in Figure 1a, in a 2032 coin cell, a 2 mm diameter hole was made on the coin cell case and a $5 \mathrm{~mm}$ diameter glass window was glued on the case to provide a clear aperture for optical characterization while maintaining a hermetic seal. In order to observe color change at the graphite particles, a hole of $500 \mu \mathrm{m} \times 500 \mu \mathrm{m}$ was cut in the copper foil with a razor blade. The electrode was assembled into a cell with electrolyte consisting of $\mathrm{LiPF}_{6}$ in carbonate solvents, porous polyolefin separator, and lithium metal counter-electrode inside a dry room. The microscope views the graphite adjacent to the foil, i.e., the "back" of the porous electrode. Because of the relatively high electronic conductivity of graphite, the in-plane ohmic drop across the hole would not significantly affect the observations.

The in-situ cell was placed into a printed-circuit board (PCB) coincell holder, and then it was fixed on the sample stage of an optical microscope at $50 \times$ magnification (Micro support, Axis pro) as shown in Figure 1b. The PCB holder was connected to a potentiostat (BioLogics, Bi-stat) for electrochemical cycling.

The cell went through formation cycles ending with complete discharge. As will be shown below, interesting phenomena are observed during rest. Therefore, we focus our attention on pulse chargedischarge behavior. Figure 2 shows the measured cell voltage and state of charge during the charge-discharge protocol used during the in situ optical experiment and in the simulations below. $1 \mathrm{C}$ rate corresponds to $350 \mathrm{mAh} / \mathrm{g}$.

The fully delithiated electrode is gray, as shown in Figure 3(left). During the $0.5 \mathrm{C}$ lithiation, it gradually turns a uniform blue. By 0.87 $\mathrm{h}$ as shown in Figure 3(right), areas of purple are forming at particle edges. Note that, since the electrodes used were quite thick and dense, the reaction rate starts out much higher closer to the separator than at the current collector. Since the microscope views the region adjacent

\section{Table I. Stages in lithiated graphite at room temperature.}

$\begin{array}{ccc}\mathrm{x} \text { in } \mathrm{Li}_{\mathrm{x}} \mathrm{C}_{6} & \text { color } & \text { stage } \\ 0 & \text { grey } & 1 \mathrm{~L} \\ 0.12 & \text { blue } & 4 \mathrm{~L} \\ 0.21 & \text { purple } & 3 \mathrm{~L} \\ 0.5 & \text { red } & 2 \\ 1.0 & \text { gold } & 1\end{array}$

From Ref.3. "L" indicates "liquid-like" stage, in which lithium occupies random positions within its layer. 

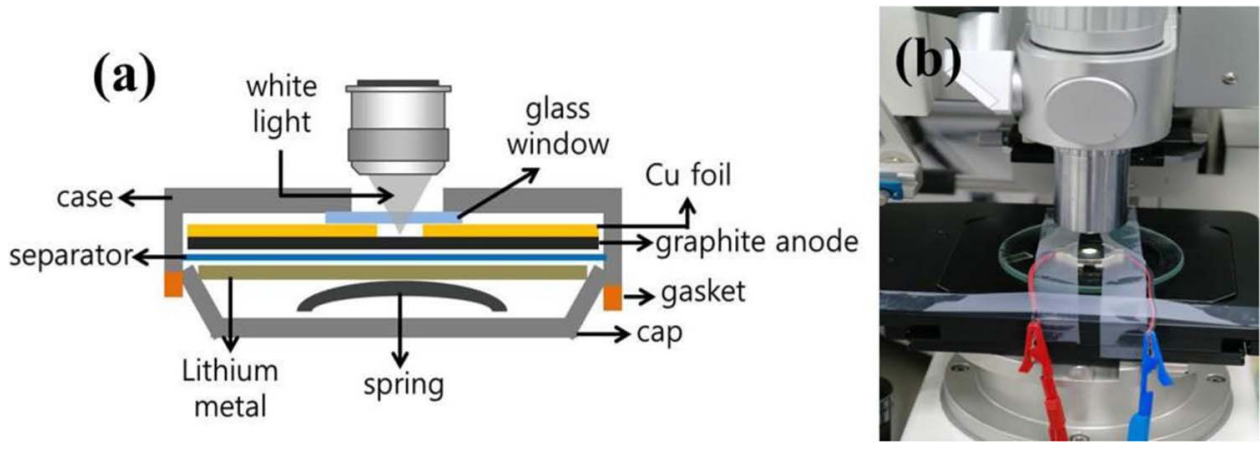

Figure 1. (a) Side view schematic of coin type half-cell for in-situ optical measurement, and (b) experimental set-up for in-situ optical measurement during cell cycling.

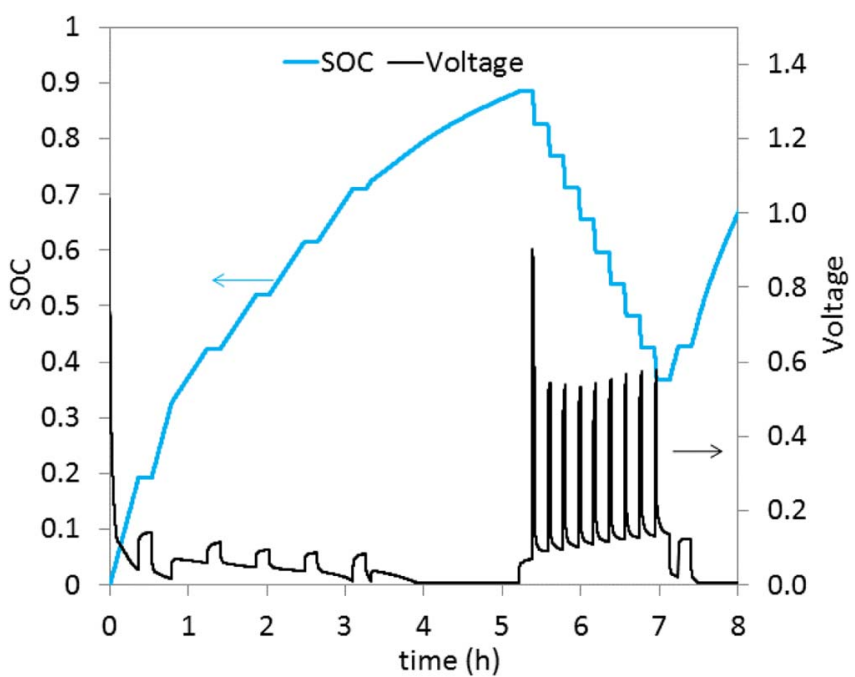

Figure 2. Cell state of charge and voltage during the in situ experiments and model simulations. The cell was lithiated at the $0.5 \mathrm{C}$ rate with a rest at $20 \%$ SOC, then lithiated at $0.2 \mathrm{C}$ with rests every 27 minutes, followed by $\mathrm{CV}$ hold at $0.005 \mathrm{~V}$. The cell was then pulse delithiated at the $2 \mathrm{C}$ rate for $1.6 \mathrm{~min}$ with 10 minute rests until it reached $37 \%$ SOC, after which the cell was lithiated at $0.5 \mathrm{C}$, ending with CV hold at $0.005 \mathrm{~V}$.

to the current collector, the local concentration seen in the particles (purple corresponding to $\sim 0.21$ ) is lower than the average state of charge across the electrode $(34 \%$ at $0.87 \mathrm{~h})$. In this paper, we use percent to indicate average state of charge across the cell and decimal numbers to indicate local concentration.

At $1.12 \mathrm{~h}$, the transition to stage 2 (concentration of 0.5 ) begins. Figure 4 shows the image after lithiation to $52 \%$ SOC. The image on the left was taken shortly before turning off the current. The image on the right was taken after 10 minutes of rest. In agreement with

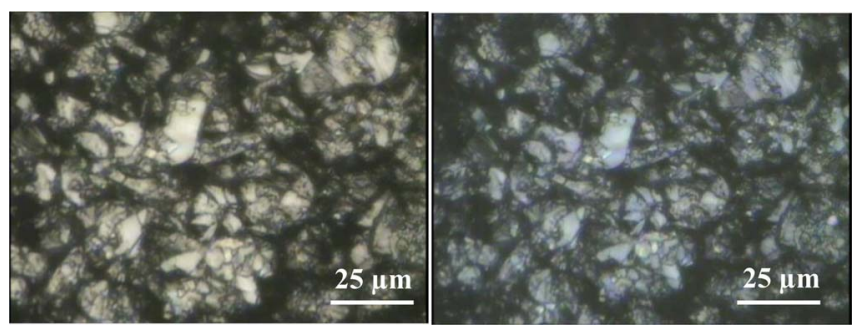

Figure 3. (left) Image at time 0 , when the electrode is fully delithiated. (right) At time $0.87 \mathrm{~h}$ (34\% SOC), color is changing from blue to purple. The field of view is $96 \times 128 \mu \mathrm{m}$.
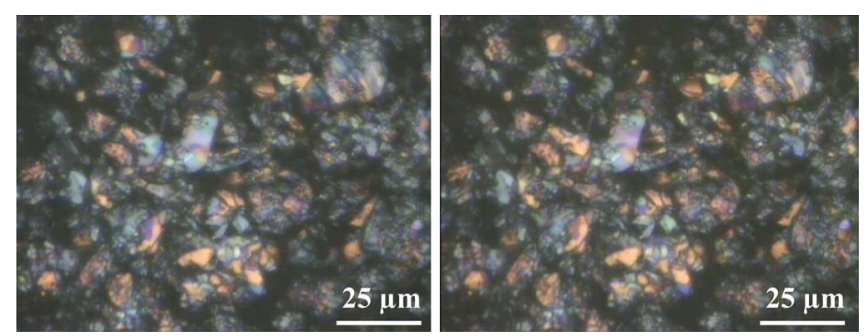

Figure 4. Image at end of lithiation to $52 \% \mathrm{SOC}$ at $1.85 \mathrm{~h}$ (left) which is then followed by 10 minutes of rest (right, at $2.02 \mathrm{~h}$ ).

the stage transitions observed via $\mathrm{XRD},{ }^{3}$ we see co-existence of red and purple stages, with no relaxation of red to purple even after 10 minutes of rest. This observation was repeated during every period of rest: there is no relaxation to uniform concentration within particles during rest; instead, stages coexist, e.g., purple coexisting with red, or red coexisting with gold. There is a small increase in red color after 10 minutes because of redistribution from the front to the back of this thick electrode. We also observe considerable spatial nonuniformity, with different colors within even what appear to be regions of the same secondary particle.

After the back of the electrode has completely converted to the red phase, the gold phase nucleates at the edges of the particles, moving across the particles until the entire electrode is uniformly gold. After holding at $0.005 \mathrm{~V}$ for 77 minutes, followed by 10 minutes of rest, the cell begins pulse delithiation at the $2 \mathrm{C}$ rate. The high current exacerbates the current nonuniformity across the thick electrode, so that the color change at the region viewed by the microscope lags the average state of charge of the electrode. Figure 5 contrasts the appearance of the electrode lithiated at low rate to $76 \%$ average state of charge, to the appearance of the electrode delithiated at high rate to $54 \%$ SOC. The stage 2 color is a lighter shade of red, and the red-gold boundary is more distinct, on low-rate lithiation than on high-rate delithiation. Further experiments are needed to understand these observations.
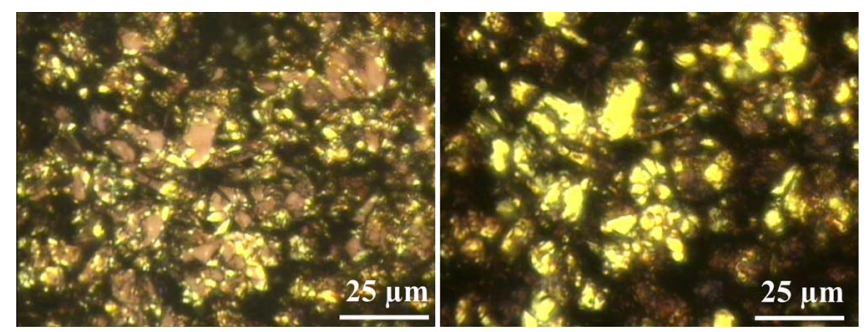

Figure 5. (left) time $3.67 \mathrm{~h}, 0.2 \mathrm{C}$ lithiation to $76 \%$ SOC. (right) $6.38 \mathrm{~h}, 2 \mathrm{C}$ delithiation to $54 \%$ SOC. 

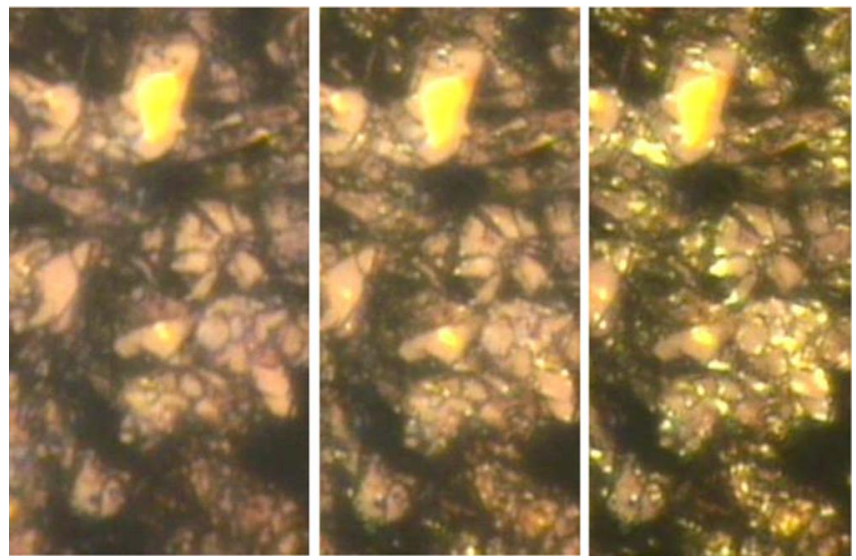

Figure 6. From left to right, images take at $7.13 \mathrm{~h}$ (after rest after delithiation to $37 \% \mathrm{SOC}$ ), $7.43 \mathrm{~h}$ (during lithiation), and $7.7 \mathrm{~h}$ (continued lithiation). We see stage 1 (gold) in the center of particles delithiated to $37 \%$ SOC, coexisting with stage 2 (red). Upon lithiation, stage 1 nucleates at the edges of the particles, while stage 1 continues to exist in the center of the particle.

The graphite was then pulse delithiated to $37 \%$ SOC, rested for 10 minutes, and then lithiated. Figure 6 shows images from three points in time. The left image shows the electrode after rest at $37 \%$ SOC after delithiation. Since the cell is at an intermediate SOC, interior regions of the particles are still gold, while the exterior regions are red. The middle image shows the electrode when it is just beginning to lithiate. Nucleation of regions of gold is clearly seen at the particle edges, while there is no change in the area of the interior gold regions. The right image was taken after further lithiation has occurred. It shows movement of the gold phase front from the edges into the interior of the particles.

\section{Model}

The electrochemical model applies the framework developed by Newman, ${ }^{8-10}$ including porous electrode theory to describe the current balance, concentrated solution theory to describe ion concentration and potential in the electrolyte, Ohm's law in the bulk electronically conducting phases of the electrodes, and Butler-Volmer kinetics with film resistance from the solid-electrolyte interphase (SEI). This modeling framework is extended to a phase-field model of transport within particles considering the stage transitions occurring within the graphite.

The phase-field model for graphite is described in detail in Ref. 11. Previous descriptions of phase-field theory are provided in Refs. 12-15. In brief, the flux of lithium within the graphite particles is proportional to the gradient in chemical potential:

$$
\text { Flux }=-\frac{D}{R T} c_{s} \nabla \mu
$$

Chemical potential (see appendix A) is the variational derivative of free energy $G$ with respect to concentration:

$$
\mu=\frac{\delta G}{\delta c_{s}}=\mu\left(c_{s}, \nabla^{2} c_{s}\right)
$$

Free energy is a function of both concentration and gradients of concentration. Where common tangent lines can be constructed under the free energy vs. concentration function, the system can lower its energy by phase separating. Within this region it can remain homogeneous in a metastable state as long as it is outside the smaller region of negative curvature, within which spinodal phase separation occurs. We apply a free energy model ${ }^{11}$ that yields an open-circuit voltage (see Figure 7) that matches the sloping voltage at low concentration as well as the stage transitions occurring from 0.21 to 0.5 and from 0.5 to 1.0 . Unlike the free-energy model used in a recent phase-field model of graphite which used two lithium concentration variables for

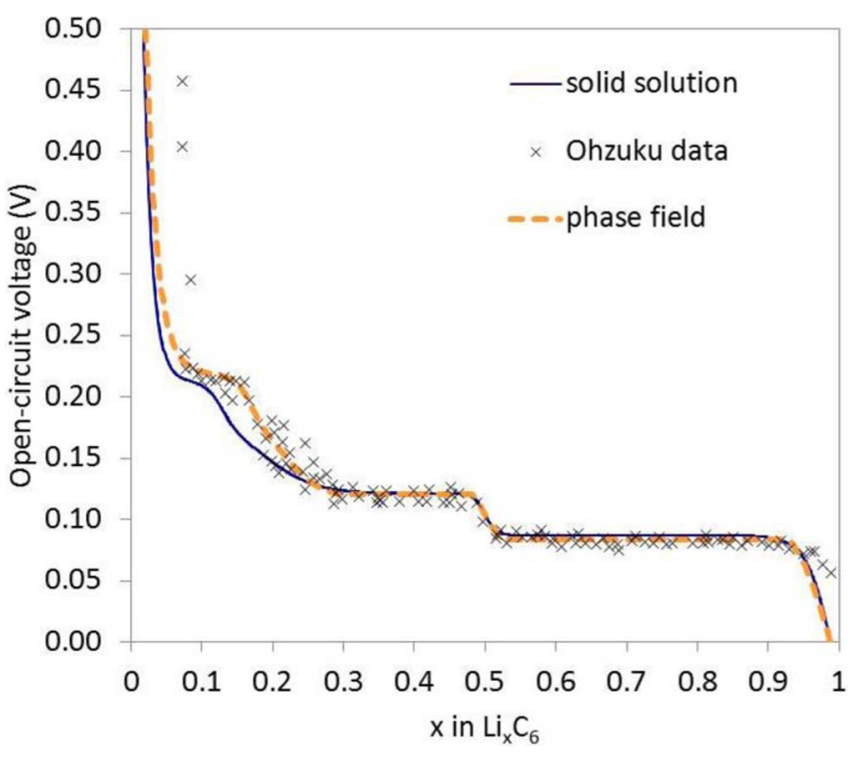

Figure 7. Open-circuit voltage as a function of lithium concentration used for both the solid solution (solid line) and phase-transition (dashed line) models. Data points are from Ref. 36.

periodically repeating layers, ${ }^{16,17}$ this model reproduces the experimental open circuit voltage with a single concentration variable and three free-energy minima.

Several previous studies have modeled graphite using equations that describe diffusion in a solid solution. ${ }^{5-7}$ In a solid-solution model, the chemical potential is a unique function of concentration only (not gradients of concentration), and Equation 1 is replaced with Fick's first law of diffusion:

$$
\text { Flux }=-D \nabla c_{s}
$$

Below, we assess the implications of the model formulation.

Parameter values used in the simulations are shown in Table II. The model described by ${ }^{11}$ was modified as follows to better match the compendium of observed behavior of graphite. The diffusivity in the graphite and the SEI film resistance on the lithium counter-electrode and on the graphite were fit to the cell data. A diffusivity that varied with concentration was considered; however, a constant diffusivity in the graphite was found to give the best match to the voltage transients for both models. It is worth noting that with the solid-solution model, a constant diffusivity enables use of Duhamel's superposition integral for calculation of the concentration within the graphite particles, thus reducing the model from a pseudo $2-\mathrm{D}$ model to a $1-\mathrm{D}$ model without

\begin{tabular}{|c|c|c|c|}
\hline Parameter & Unit & Negative & Separator \\
\hline thickness & $\mu \mathrm{m}$ & 97 & 25 \\
\hline porosity & & 0.258 & 0.40 \\
\hline volume fraction & & 0.039 & \\
\hline binder + conductive & & & \\
\hline particle radius & $\mu \mathrm{m}$ & 8 & \\
\hline $\begin{array}{l}\text { bulk electronic } \\
\text { conductivity }\end{array}$ & $\mathrm{S} / \mathrm{m}$ & 50 & \\
\hline $\begin{array}{l}\text { tortuosity in } \\
\text { electrolyte }\end{array}$ & & $2 \varepsilon^{-0.5}$ & $\varepsilon^{-0.5}$ \\
\hline Parameter & Unit & Graphite & Lithium \\
\hline diffusivity & $\mathrm{m}^{2} / \mathrm{s}$ & $\begin{array}{l}1 \times 10^{-14} \text { (solid solution) } \\
0.4 \times 10^{-14} \text { (phase field }\end{array}$ & \\
\hline $\begin{array}{l}i_{0} \text { at } 1 \mathrm{M}, 50 \% \text { SOC } \\
\text { SEI film resistance }\end{array}$ & $\begin{array}{l}\mathrm{A} / \mathrm{m}^{2} \\
\text { ohm- } \mathrm{m}^{2}\end{array}$ & $\begin{array}{c}40 \\
0.001\end{array}$ & $\begin{array}{c}800 \\
0.0025\end{array}$ \\
\hline
\end{tabular}

Table II. Geometric and material properties used in the simulations. 


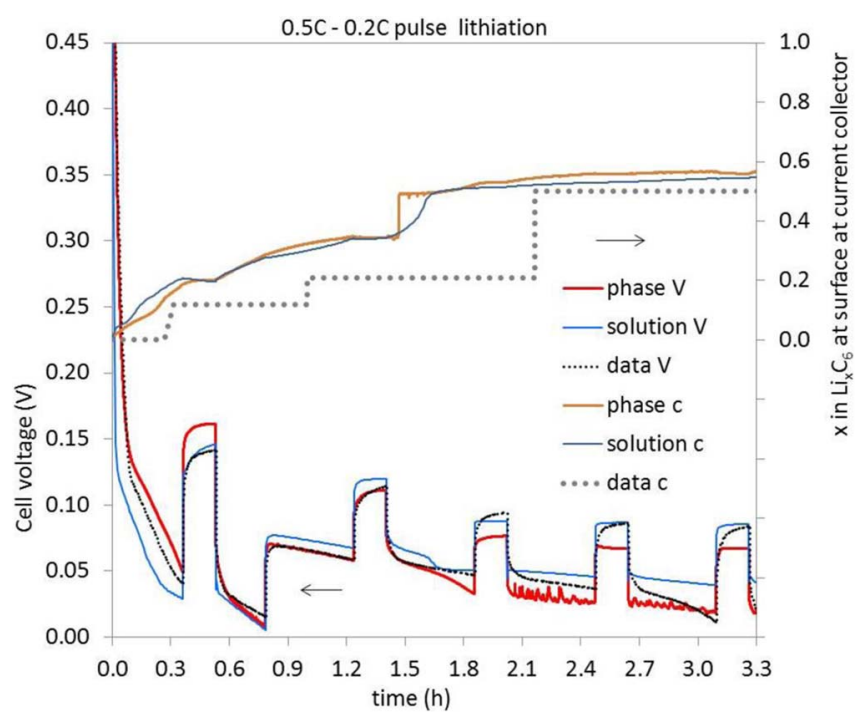

Figure 8. Comparison of experimental data during pulse lithiation to simulated voltage and concentration (at particle surface at the position of the current collector), for both the solid-solution ("solution") and the phase-transition ("phase") models.

any loss of fidelity. ${ }^{18,19}$ This model reduction reduces the computation time by roughly two orders of magnitude.

To match the dependence of charge-transfer resistance on current and on state of charge, we include a SEI film resistance (which is constant with current and state of charge) in the Butler-Volmer equation for both graphite and lithium metal: ${ }^{8}$

$$
\begin{aligned}
i_{n}= & i_{0}\left[\exp \left(\frac{\alpha_{a} F}{R T}\left(\phi_{1}-\phi_{2}-U-R_{f i l m} i_{n}\right)\right)\right. \\
& \left.-\exp \left(\frac{-\alpha_{c} F}{R T}\left(\phi_{1}-\phi_{2}-U-R_{f i l m} i_{n}\right)\right)\right]
\end{aligned}
$$

The SEI film resistance on the lithium metal comprises the majority of the total resistance of the cell, and the SEI film resistance on the graphite comprises the majority of the charge-transfer resistance on the graphite. The exchange-current density for graphite varies with both electrolyte and solid concentrations according to:

$$
i_{0}=k\left(\frac{c}{c_{r e f}}\right)^{\alpha_{c}}\left(\frac{c_{s}}{c_{\max }}\right)^{\alpha_{a}}\left(\frac{c_{\max }-c_{s}}{c_{\max }}\right)^{\alpha_{c}}
$$

with $\alpha_{\mathrm{a}}=\alpha_{\mathrm{c}}=0.5$ and $c_{\text {ref }}=1 \mathrm{M}$.

We use the electrolyte diffusivity, transference number, and activity coefficient measured by Valoen and Reimers, ${ }^{20}$ and the conductivity measured by Bernardi and Go. ${ }^{21}$ Tortuosity is estimated ${ }^{22}$ to be $2 \varepsilon^{-0.5}$, which we have found to match experimentally-measured tortuosity in electrodes over typical ranges of volume fractions of porosity and binder for commercial graphite electrodes.

The simulations used 20 mesh points across the porous electrode and 40 radial mesh points within the spherical particles. When considering spatial variability below, multiple particle types are considered at each location across the porous electrode; for all other simulations shown in this paper, the simulations use a single particle type at each location. The solid-solution model was based on dualfoil.f which uses finite differences with control volumes, solved via $\operatorname{BAND}(\mathrm{j}) .^{23}$ The phase-field model, named mpet.py, uses finite volumes, solved via DAE Tools. $^{24}$

\section{Comparison of Model to Experimental}

Figures 8 and 9 show the comparison of both the phase-transition and the solid-solution model to the experimental data. We see that both models are able to match the experimentally observed voltage

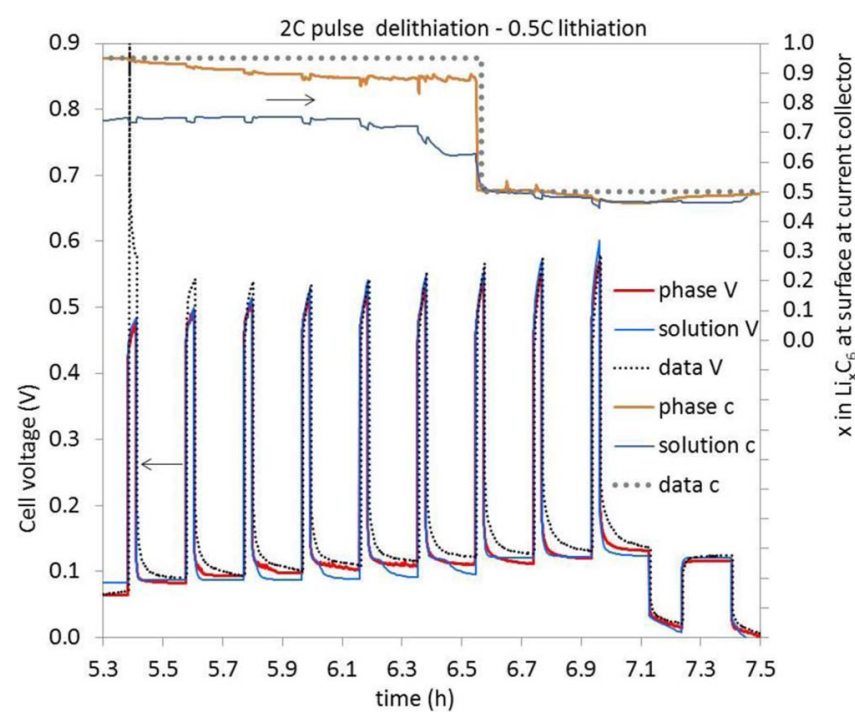

Figure 9. Comparison of experimental data during high-rate pulse delithiation, followed by lithiation, to simulated voltage and concentration (at particle surface at the position of the current collector) for both the solid-solution and the phase-transition models.

transients. It is not clear why the data show a voltage spike during the first 2C-rate pulse delithiation; most likely it arises from the lithium counter electrode. Also shown is the solid concentration. We plot the simulated concentration at the particle surface at the position adjacent to the current collector, as that position is what is observed in the experiments. The spatial mode (most common value) of the experimental color data is converted to numerical concentration based on the calibration taken from the literature in Table I. The difference between the simulated concentrations is most evident between 6.3 and $6.5 \mathrm{~h}$. The solid-solution model allows the concentration to vary continuously between 0 and 1 , and thus gives surface concentrations between 0.7 and 0.8 during this time. In contrast, the phase-transition model allows only small deviations from the stage compositions of 1.0 and 0.5 . When the cell is at an intermediate state of charge, the particles contain regions of 0.5 and 1.0 compositions. The solid-solution model shows relaxation of the concentration during rest between 6.4 and 6.5 $\mathrm{h}$, whereas the phase-transition model shows continued existence of the phase at the surface of the particle during rest. We consider the implications of this difference in Lithium plating section.

As described in Experimental methods and observations section, the experimental data show 1) that the observed concentration lags the state of charge (i.e., average concentration across the electrode), and 2) there is a small change of concentration during rest to higher (following lithiation) and lower (following delithiation) states. To understand these observations, Figure 10 compares the simulated concentrations at the particle surfaces at two positions in the cell: adjacent to the current collector ("back") and adjacent to the separator ("front"). The in situ experiments view the position adjacent to the current collector. However, the local reaction rate is initially highest adjacent to the separator, because the effective ionic conductivity of the electrolyte $(0.1 \mathrm{~S} / \mathrm{m})$ in the porous electrode is substantially lower than the electronic conductivity of the bulk electrode $(50 \mathrm{~S} / \mathrm{m})$. The higher local reaction rate is indicated by the more rapid change in concentration when current is applied. This nonuniform reaction-rate distribution explains why the observed concentration at the back of the electrode significantly lags the state of charge, particularly at higher current, as observed in Figure 5. When the current is turned off, internal reaction occurs within the electrode because of differences in electrochemical potential across the electrode. ${ }^{25}$ For example, during rest following lithiation at $1.24 \mathrm{~h}$ and also at $1.86 \mathrm{~h}$ in Figure 10, this internal reaction leads to a decrease of the concentration at the front, accompanied by 


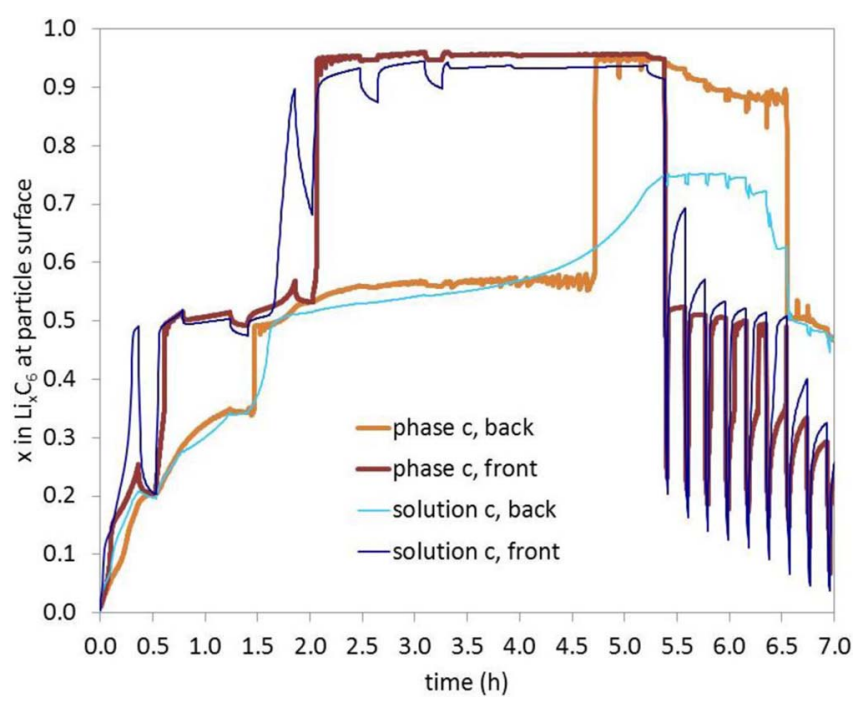

Figure 10. Simulated surface concentration at both the front (adjacent to separator) and back (adjacent to current collector). The concentration changes more rapidly at the front because of the high resistance in the electrolyte in this thick, dense electrode. During rest, internal reaction redistributes lithium from the front to the back of the electrode.

increase in concentration at the back, in agreement with the experimental observations (see Figure 4).

Figure 11 compares the radial concentration profiles within particles, for the time period corresponding to Figure 6 . These time steps, comprising periods of rest and change in direction of current at intermediate states of charge, were selected to highlight the main difference between the models. The phase-transition model predicts coexistence of phases at equilibrium, whereas the solid-solution model predicts relaxation of concentration gradients during rest. The phase-transition simulation captures the core-shell-shell distribution seen in Figure 6.

While the models differ in their treatment of diffusion within particles, they show similar profiles for the reaction-rate distribution across the electrode. This is because we have chosen the open-circuit potential function for the active material in the solid-solution model to have a flat plateau during compositions corresponding to two-phase transi- tions. Figure 12 shows the particle surface concentration distribution across the porous electrode, at time steps corresponding to the end of lithiation, end of delithiation, and during rest. When the current is on, ohmic drop in the electrolyte causes the reaction rate to be higher closer to the separator, leading to gradients in the lithium concentration across the electrode. During rest, gradients in chemical potential can cause internal current, relaxing the concentration gradient. However, if the chemical potential is constant across a range of concentration, i.e., when there is a horizontal plateau in the open-circuit voltage profile, then there is no driving force for relaxation of concentration gradients. In contrast, even a slight slope in the open-circuit profile would cause a driving force for relaxation across the electrode, with a higher slope providing a higher driving force for relaxation. Since the open-circuit potential is constant over the concentration range from 0.55 to 0.95 , the solid-solution model shows a concentration of about 0.75 in the back of the electrode even though the concentration at the front of the electrode varies over extended time from 0.7 to 0.93 . In the phase-transition model, the curves during rest overlie the curves at the end of current steps, because the surface concentration stays within the range of phase coexistence. Thus, both models can show gradients in concentration across the porous electrode, because internal relaxation is driven by chemical potential and both have similar dependence of chemical potential on concentration.

\section{Spatial Distribution}

Figure 4 shows considerable spatial nonuniformity in the concentration distribution. In this section, we consider whether this amount of nonuniformity is consistent with known causes of spatial nonuniformity. First, we consider known causes of spatial nonuniformity of electrochemical resistance. Then, we implement a cartoon representation of these phenomena. We run simulations with different magnitudes of spatially varying resistance until the simulated spatial variation matches that observed experimentally. Finally, we assess whether the magnitude of the resistance necessary to cause such spatial variation is consistent with known phenomena.

Several parameters are known to vary spatially:

1. Tortuosity, i.e., the ratio of actual path length to apparent path length, varies across a packed bed of irregularly-shaped, variously-sized particles. There may be a more direct path through the electrolyte to access the surface of some particles, whereas

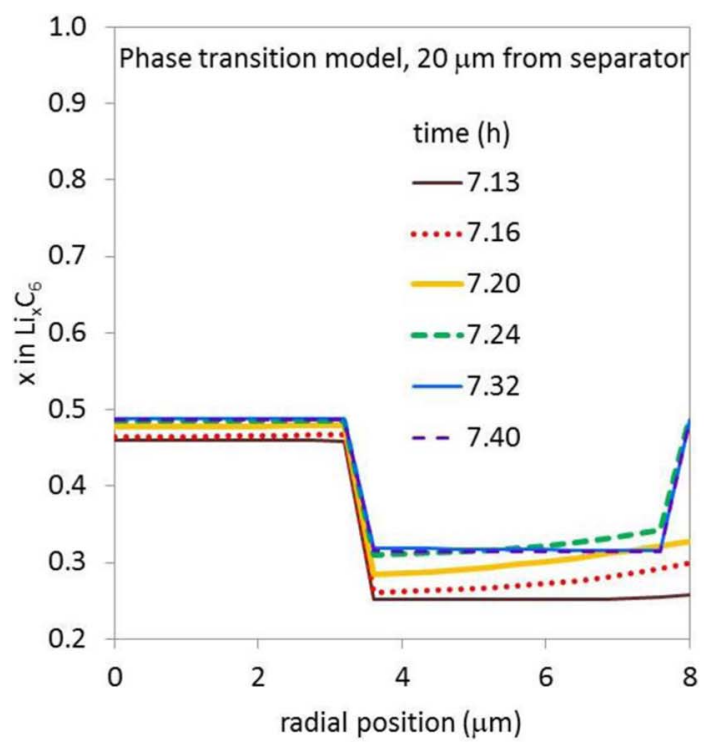

Figure 11. Simulated radial concentration profiles from the phase-transition model (left) and solid-solution model (right), at position $20 \mu \mathrm{m}$ into the electrode. The center of the particle is at radial position 0. Prior to the time steps shown, the electrode has undergone full lithiation, partial delithiation, and then rest. The time steps shown correspond to end of rest (7.13 h), partial lithiation (ending at $7.24 \mathrm{~h}$ ), and rest (ending at $7.40 \mathrm{~h}$ ). 

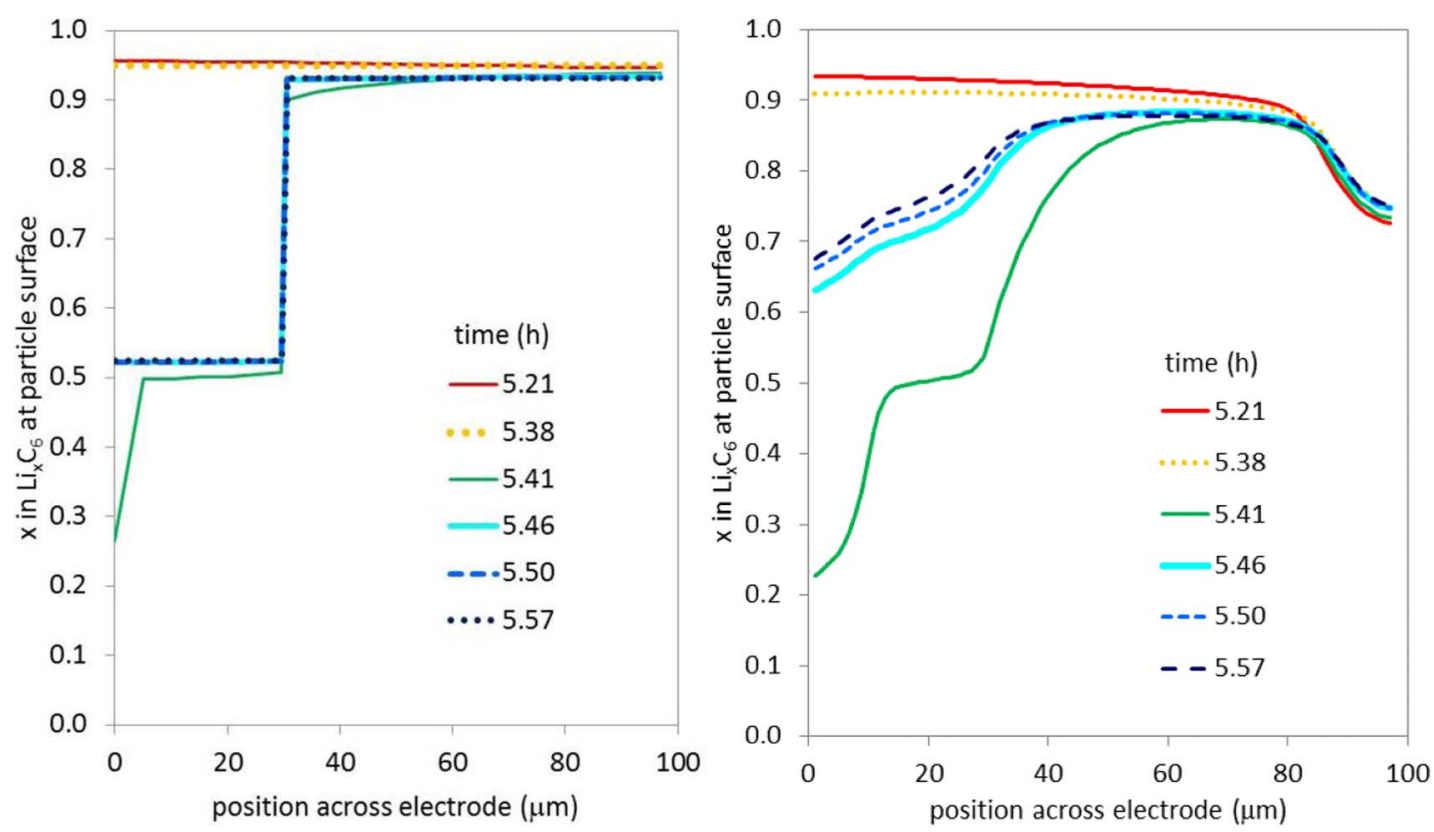

Figure 12. Simulated surface concentration profiles across the electrode from the phase-transition model (left) and solid-solution model (right). The separator is at position 0 , and the current collector is at $97 \mu \mathrm{m}$. The timesteps shown correspond to end of $\mathrm{CV}$ hold at $0.005 \mathrm{~V}(5.21 \mathrm{~h})$, end of rest $(5.38 \mathrm{~h})$, end of $2 \mathrm{C}$-rate delithiation $(5.41 \mathrm{~h})$, and end of 10 minute rest $(5.57 \mathrm{~h})$.

other particles can only be accessed by travelling through more constricted and/or circuitous pores. The ratio of the highesttortuosity path length to the lowest-tortuosity path length can be as large as 2.5. ${ }^{26}$ This difference in ionic resistance causes variation in the local reaction rate, which leads to variation in local lithium concentration in the active material..$^{22,27,28}$ The magnitude of resistance variation is approximately (pathlength difference)/ [ $\kappa^{*}$ (area difference)], which can range from $10^{3}$ to $10^{7} \mathrm{ohm}$. Normalized by the surface area of a particle, to enable comparison with the magnitude of other aspects of cell resistance such as charge transfer resistance, the magnitude of resistance variation is $10^{-5}$ to $10^{-2} \Omega \cdot \mathrm{m}^{2}$.

2. Crystallite orientation within secondary particles: Graphite diffusion is two-dimensional, with facile diffusion parallel to the basal plane but energetically unfavorable diffusion perpendicular to the basal plane. ${ }^{4}$ Commercial graphite consists of secondary particles available in average particle size of 5 to $20 \mu \mathrm{m}$, comprised of primary particles (crystallites) of 0.02 to $0.3 \mu \mathrm{m} .^{29,2}$ The basal plane of these crystallites can be randomly oriented with respect to the surface of the particle. Thus, lithium transport will be faster where particle surfaces coincide with crystallite edge planes, and correspondingly lithium transport will be slower where particle surfaces coincide with crystallite basal planes. ${ }^{30}$ Also, graphite particles with smaller crystallite size, e.g. less-graphitic artificial graphite, have more facile lithium transport than graphite with larger crystallite size, e.g. natural graphite, i.e., grain-boundary transport is faster than within-grain transport. ${ }^{29}$

3. Particle-particle contact resistance: Electronic contact resistance between particles can occur because of poor packing within the asfabricated porous electrode. ${ }^{31}$ Increase in particle-particle contact resistance is a major cause of capacity fade and impedance rise, because electronically insulating SEI grows between particles over the course of many cycles.

4. SEI film resistance: the SEI film resistance is a large component of the charge-transfer resistance and indeed of the total resistance of the graphite electrode, and its magnitude is strongly affected by electrolyte composition during cell formation. Variations in the SEI film resistance can arise from variation of surface roughness, variation in SEI conductivity and/or thickness arising from spatially nonuniform transport of SEI-forming additives during cell formation, and impurities in the cell.

5. Particle size: Smaller particles have lower resistance because of their higher surface area and shorter diffusion pathlength. However, evaluation of the in situ movies did not show a noticeable correlation between particle-size distribution and reaction rate; large particles were just as likely as small particles to transform first.

While detailed simulations of transport within the microstructure of an electrode have been carried out, ${ }^{32}$ they are computationally cumbersome and time-consuming to employ for purposes of cell and module design. For example, while the macrohomogeneous models in this paper were able to sufficiently model the electrode behavior using 800 mesh points (20 across the electrode and 40 radial within particles), a microstructural model would employ $10^{4}$ to $10^{7}$ mesh points to describe a single electrode. Our goal is the development of a model that captures the necessary physics with minimum computational time so that it can be used for engineering design and control simulations. All of the phenomena listed above can be approximated as a distributed resistance, i.e., some particles have a lower-resistance connection to the average electrode potential (because of shorter electrolyte pathway, proximity of edge planes, good electrical connection, and/or locally low SEI film resistance) while other particles have a higher-resistance connection (because of a more tortuous pathway to that particle, particle surface faced with basal planes, poor particleparticle contact, or locally high SEI film resistance).

Therefore, we consider a cartoon model with a distributed resistance. We make no assertion as to the magnitudes of the relative contributions from the different physical phenomena to the overall distributed resistance; such conclusions would require detailed experiments beyond the scope of this investigation.

The phase-transition model is expanded to include multiple particles $k$ at each mesh point across the electrode, each particle having a different contact resistance to the bulk conducting matrix, as shown schematically in Figure 13. Particle volume fraction is uniform across the particle types. The gradient in bulk potential across mesh points is governed by Ohm's law. Within a mesh point, potential differs among 


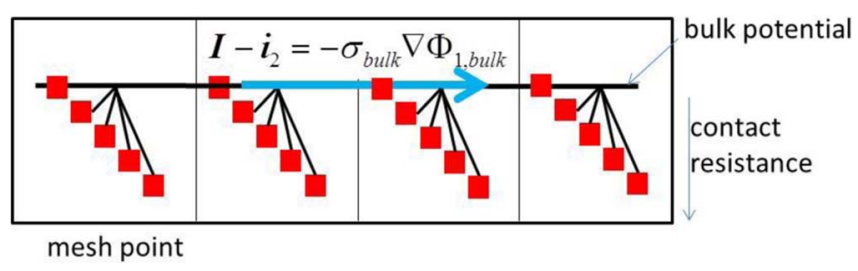

Figure 13. Schematic for contact-resistance model. Shown are four mesh points, each containing five particle types. The particle types are connected in parallel to the bulk electronic potential. Ohm's law governs the bulk potential, where $I-i_{2}$ is the current in the electronic phases, which changes across the porous electrode owing to electrochemical reaction. The potential drop between the bulk electronic potential and each particle type depends on the local reaction rate to the particle type and that type's contact resistance.

particle types because of contact resistance:

$$
\phi_{1, k}=\phi_{1, \text { bulk }}-i_{n, k} R_{\text {contact }, k}
$$

where $i_{n, k}$ is the transfer current per surface area of particle type $k$ and $R_{\text {contact, } \mathrm{k}}$ is normalized by the surface area of particle type $k$.

We then ran simulations with different magnitudes of the average contact resistance until the simulations matched the amount of spatial nonuniformity observed in the experiments. The results are shown in Figure 14. " 1 st" indicates the first point in time at which the composition is observed. "Avg" indicates the time at which half of the observed area has the indicated composition.

As shown in Figure 14, an average contact resistance of $2 \times 10^{-3}$ $\Omega \cdot \mathrm{m}^{2}$, standard deviation $1 \times 10^{-3} \Omega \cdot \mathrm{m}^{2}$, using five particle types was found to give a reasonable match to the difference in time between first appearance and average appearance of new phases. This magnitude is within the reasonable range of the combined contributions of tortuosity, crystallite orientation, particle-particle contact resistance, and/or variable SEI film resistance.

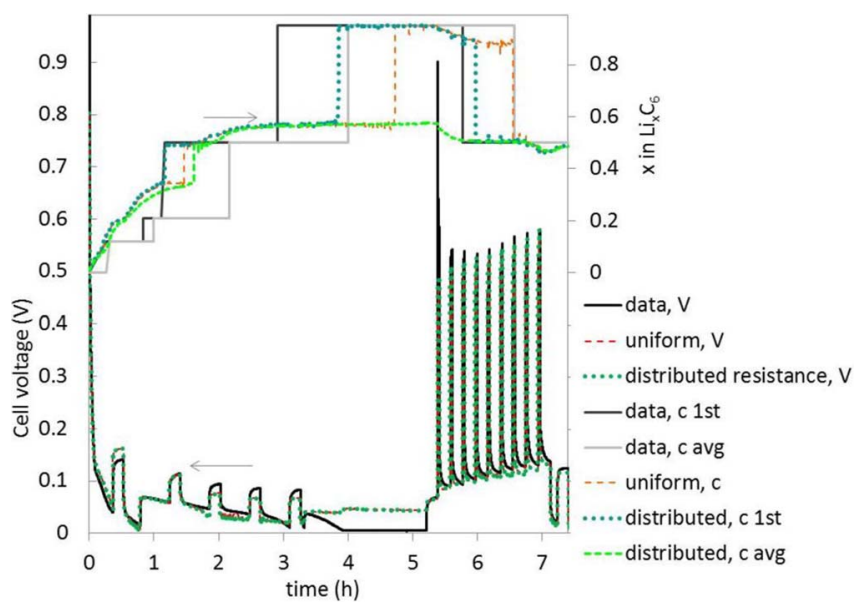

Figure 14. Simulated and measured cell voltage and concentration at the position of the current collector, for the same electrochemical experiments described in Figure 2. Two simulations are shown; with uniform particles (which was also shown previously in Figure 8), and with a distribution of local resistances within each mesh point. The mean of the distributed resistances is $0.002 \Omega \cdot \mathrm{m}^{2}$. For the simulation with a distribution of resistances and for the experimental data, two concentration profiles for each are shown: "first appearance", i.e., the first observation of the indicated phase (which occurs at the particle with zero resistance to the conducting bulk in the simulation), and "average appearance", i.e., when half of the observed area has transitioned to the indicated phase (which occurs at the particle with highest resistance to the conducting bulk in the simulation).

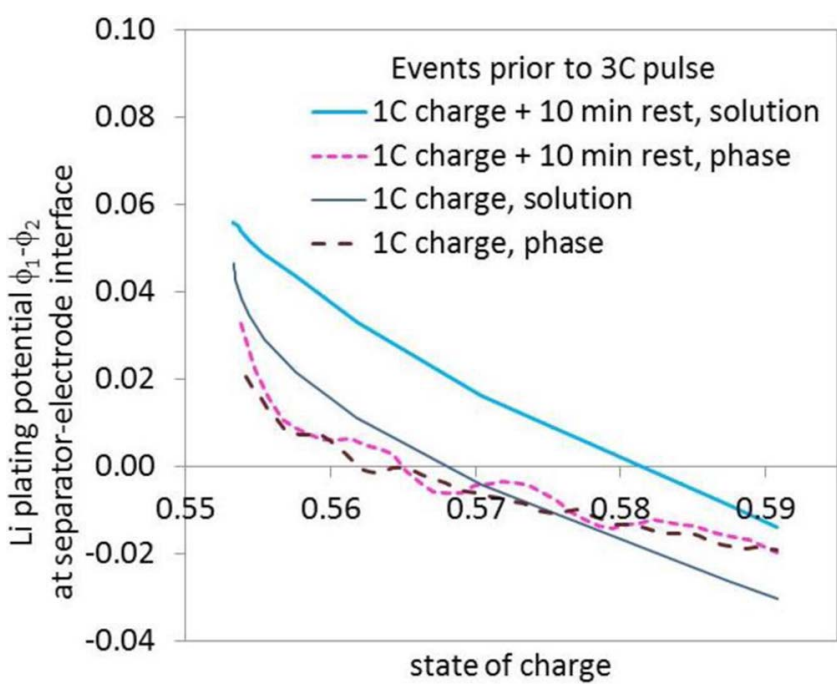

Figure 15. Simulated lithium plating potential during a 3C-rate charge from 55 to $59 \%$ SOC, comparing the effects of events preceding this 3C-rate charge. Darker lines: before the 3C-rate pulse, the cell was charged at 1C to 55\% SOC. Lighter lines: before the 3C-rate pulse, the cell was charged at $1 \mathrm{C}$ to $55 \%$ SOC then allowed to rest for 10 minutes. Solid lines: solid-solution model. Dashed lines: phase-transition model.

\section{Lithium Plating}

Phase transitions.-One of the main degradation mechanisms in lithium-ion batteries is lithium plating, which can occur when the battery is charged too quickly, particularly as the battery ages. Lithium plated on the graphite can react with the electrolyte to form inactive compounds, leading to loss of cyclable lithium and increased resistance. Because of the high importance of accurate prediction of the risk of lithium plating, we explore the difference in the predicted risk of lithium plating between the phase-transition and the solid-solution models.

The reduction reaction to deposit lithium metal is possible when

$$
\phi_{1}-\phi_{2}-i_{n} R_{f i l m}-U_{L i} \leq 0
$$

where $U_{\mathrm{Li}}=0$ since the model defines the potentials of the active materials with respect to a lithium reference electrode. For simplicity, in the following graphs we plot $\phi_{1}-\phi_{2}$, which we term the "lithium plating potential", which gives a conservative estimate of the risk of lithium plating and does not affect the conclusions regarding the two models.

Note that concentration does not appear explicitly in Equation 7. Solid-phase concentration affects the risk of lithium plating only to the extent that it affects the electrochemical potential. Potential is the driving force for reaction in both the solid-solution and the phasetransition models. Since both models employ very similar dependence of chemical potential on lithium concentration (see Figure 7), we see below that, under many situations, they give similar predictions for the risk of lithium plating.

Figures 15 and 16 show simulated results during a 3C-rate charge for $45 \mathrm{~s}$ starting from $55 \%$ SOC. Figure 15 shows the simulated lithium plating potential for the two models for two cases: continuous charge, in which the 3C-rate charge was preceded only by $1 \mathrm{C}$-rate charge to $55 \%$ SOC, and interrupted charge, in which the 3C-rate charge was preceded by $1 \mathrm{C}$ charge to $55 \%$ SOC and then 10 minutes of rest. These conditions were selected because they show the greatest differences between the models. As noted above, the main difference between the two models is the radial concentration distribution within particles during rest. The radial concentration profiles are shown in Figure 16. The solid-solution model allows radial concentration gradients to relax to a uniform concentration, with the effect that introducing a long rest period between charging events reduces the surface concentration, which delays the risk of lithium plating. 


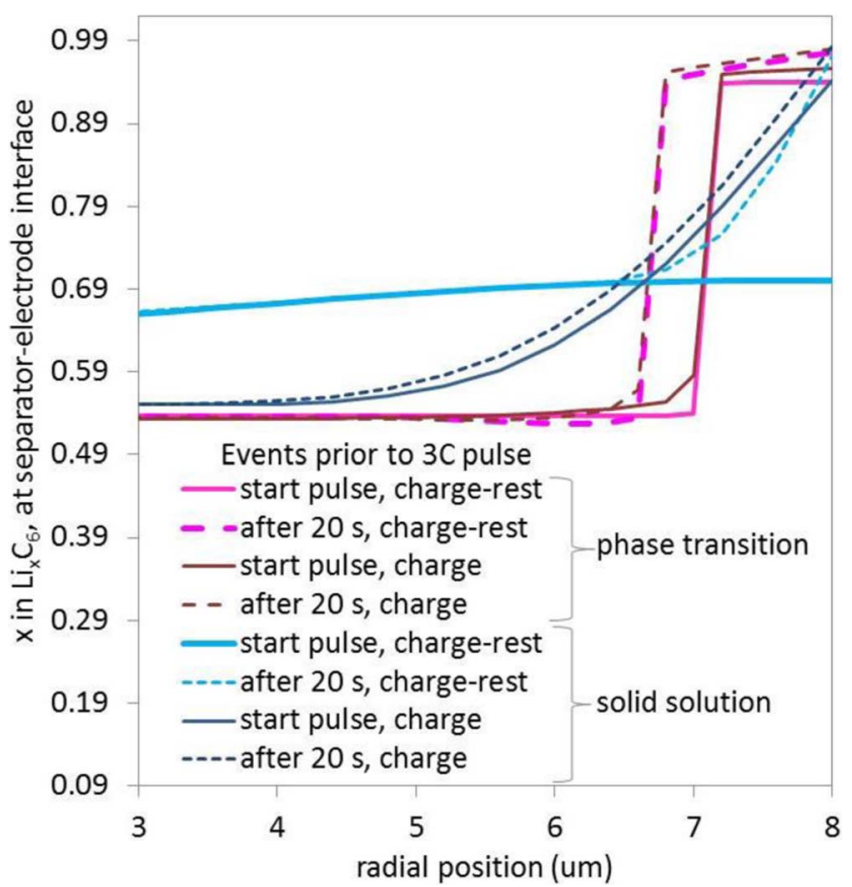

Figure 16. Simulated lithium distribution within a particle located near the separator. Simulations are shown for both the phase-transition model and the solid-solution model. The simulations show pulse time at $0 \mathrm{~s}$ and at $20 \mathrm{~s}$ during $3 \mathrm{C}$-rate charging starting at 55\% SOC, and compare the effects of events occurring prior to this $3 \mathrm{C}$-rate charging. Darker lines: the 3C-rate pulse was preceded by $1 \mathrm{C}$ charge to $55 \%$ SOC. Lighter lines: before the 3C-rate pulse, the cell was charged at $1 \mathrm{C}$ to $55 \%$ SOC then allowed to rest for 10 minutes.

In contrast, since phase boundaries do not move within particles during rest in the phase-transition model, rests affect the risk of lithium plating within the phase-transition model only to the extent that they relax electrolyte-phase concentration gradients across the electrode. For the case of continuous current (no rest period), the two models give similar predictions for the risk of lithium plating.

Spatial nonuniformity.-Next we consider the impact of spatial nonuniformity on the risk of lithium plating. We employ the same distributed-resistance model used for Figure 14, i.e., five particle types at each mesh point, each particle type having the same volume fraction, with particle-to-bulk-potential resistance randomly assigned with an average of $0.002 \Omega \cdot \mathrm{m}^{2}$ ranging from 0 to $0.02 \Omega \cdot \mathrm{m}^{2}$. The distributedresistance model is compared to the case of uniform particles, i.e., all particles have zero particle-to-bulk-potential resistance. We consider the impact on the risk of lithium plating during a stepped charging sequence, in which the cell is charged at the $2 \mathrm{C}$ rate to $50 \% \mathrm{SOC}$, then at the $1.5 \mathrm{C}$ rate to $70 \%$, and finally at the $1 \mathrm{C}$ rate to $90 \%$ SOC. The lithium plating potential is shown in Figure 17. The lithium plating potential falls below zero sooner when there is a distributed resistance. Figure 18 shows why a distributed resistance increases the risk of lithium plating. It shows the concentration at the particle surface at the position most at risk of lithium plating (nearest to the separator), for both the lowest-resistance particle type and the highestresistance particle type. When some particles have a higher resistance than others, a larger potential needs to be applied in order to drive the current through the electrode. Particles that are well-connected will react first. Reaction of the higher-resistance particles requires applying a larger driving force, which can be so large that it drives the well-connected particles below the lithium plating potential.

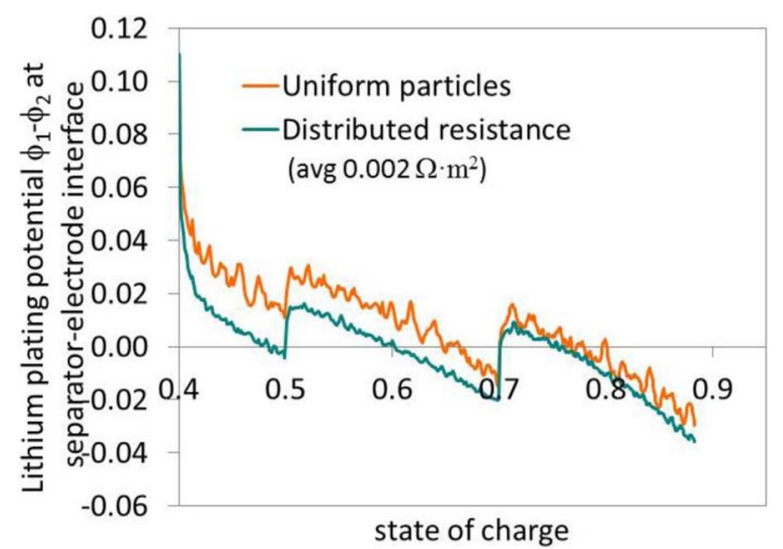

Figure 17. Lithium plating potential during charging at $2 \mathrm{C}$ to $50 \% \mathrm{SOC}$, then $1.5 \mathrm{C}$ to $70 \% \mathrm{SOC}$, then $1 \mathrm{C}$ to $90 \%$ SOC, comparing the simulated behavior of uniform particles to the behavior of the distributed resistance model.

\section{Discussion}

In addition to the two models discussed, other models for phase transitions have been proposed. A moving-boundary model (also referred to as shrinking-core model $)^{33,34}$ will give the same results as the phase-field model described here for continuous charge or discharge. However, it is difficult to formulate moving-boundary models that can accommodate the growth of an initially unknown number of phase regions within a particle as would occur during intermittent charge-discharge. For example, Figure 6 shows three regions after our relatively simple charge-discharge protocol; more regions could form during more complex operation such as is found in hybrid-vehicle operation. The phase-field model relieves the need know the number of phase boundaries in advance of running the simulation.

It has also been suggested ${ }^{33}$ that a phase transition can be approximated by a solid-solution model using the gradient of chemical potential as the driving force for diffusion, instead of the gradient of concentration. Mathematically this is equivalent to using a variable diffusivity with the gradient of concentration, with the diffusion coefficient proportional to the derivative of the activity coefficient $f$ with respect to concentration. Over the concentration range of a phase transition, $\mathrm{d} f / \mathrm{d} c$ approaches zero. Numerical stability is quite difficult when the diffusivity approaches zero, so one would choose a functional form for the activity coefficient that has a nonzero slope. This approach does allow the solid solution model to come closer to approximating the features of the phase-transition model. However, because numerical stability requires that diffusivity (and thus $\mathrm{d} f / \mathrm{d} c$ ) not be equal to zero, the model still will show relaxation of concentration gradients during rest. Also, when $\mathrm{d} f / \mathrm{d} c$ is small enough to

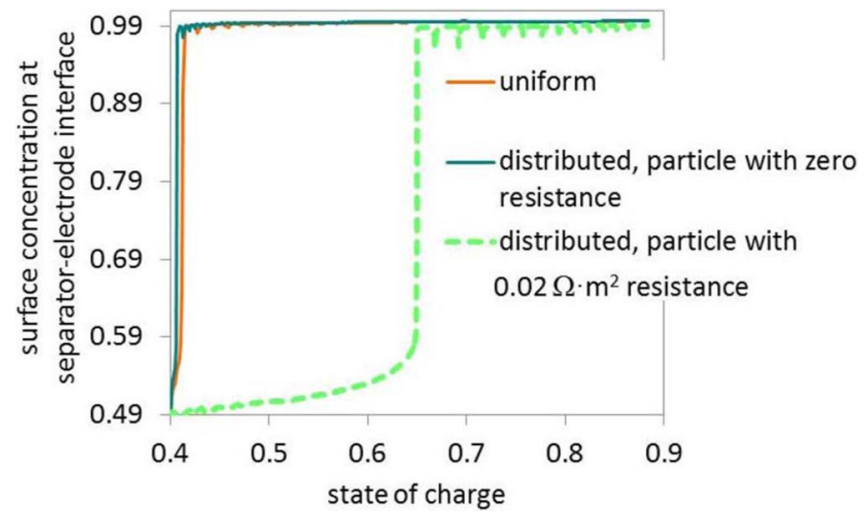

Figure 18. Surface concentration at the position adjacent to the separator, for the charging event shown in Figure 17. 
approximate the motion of a phase boundary, the diffusivity is then so low that the model will overestimate the concentration gradients at the surface of the particle.

If both the solid-solution and the phase-transition models have the same chemical potential as a function of concentration and the same diffusion time constant, as they must in order to match the experimental voltage behavior, then they will predict similar transport into the particles, i.e., similar chemical potential at the surface of particles and similar driving forces for transport at the surface of particles. The phase-transition model will provide a more accurate simulation than the solid-solution model when one is considering the impact of prolonged rest on subsequent pulse charge/discharge behavior. A key application for the phase-transition model may be for models of stress distribution within particles, because stress is generated by change in volume, which is a direct function of concentration, and therefore an accurate calculation of the concentration distribution is necessary for an accurate assessment of the stress distribution. For example, Sethuraman et al. find that the stress change with state of charge follows the stage transitions in graphite. ${ }^{35}$

\section{Conclusions}

The in situ optical experiments of the graphite electrode show that stages coexist with each other within particles, even after extended rest period. During partial depth-of-discharge cycling, complex distributions of stages can evolve within particles. We model the evolution of this stage distribution using a Cahn-Hilliard approach. The main difference in predicted electrochemical performance compared to a solid-solution model arises during rest, as the solid-solution model predicts relaxation of concentration gradients within particles, whereas the phase-transition model predicts little change in the concentration distribution within a particle during rest.

The experiments also show considerable spatial nonuniformity on the microscale. While detailed experiments and models would be needed to determine the exact sources of the spatial nonuniformity, the magnitude of spatial nonuniformity can reasonably be attributed to expected spatial variation in tortuosity, electrical contact resistance, SEI film resistance, and/or crystallite orientation. The resulting effects of these various phenomena can be approximated by a simple model with a distribution of resistances at each mesh point. The implication of a distribution of resistance among particles is that the lower-resistance particles react sooner than the higher-resistance particles, leading to higher risk of degradation, such as lithium plating, on the lowerresistance particles.

\section{Acknowledgments}

This work was supported by Samsung Advanced Institute of Technology.

\section{Appendix A}

The chemical potential in the solid for the phase field model is given by the following:

$$
\mu=0.18+\mu_{A}+\mu_{B}+\mu_{C}+\mu_{D}+\mu_{E}-\frac{\chi}{c_{\max }} \nabla^{2} \tilde{c}_{s}
$$

where $\tilde{c}_{s}=c_{s} / c_{\max }$, the gradient energy penalty term is specified by the value of $\chi=$ $4 \times 10^{-7} \mathrm{~J} / \mathrm{m}$, and

$$
\begin{gathered}
\mu_{A}=R T\left[\left(-40 \exp \left(-\frac{\tilde{c}_{s}}{0.015}\right)+0.075\left(\tanh \left(\frac{\tilde{c}_{s}-0.17}{0.02}\right)-1\right)\right.\right. \\
\left.\left.+\tanh \left(\frac{\tilde{c}_{s}-0.22}{0.04}-1\right)\right) S_{D}\left(\tilde{c}_{s}, 0.35,0.05\right)\right] \\
\mu_{B}=-R T \frac{0.05}{\tilde{c}_{s}^{0.85}} \\
\mu_{C}=10 R T S_{U}\left(\tilde{c}_{s}, 1,0.045\right) \\
\mu_{D}=6.12 R T\left(0.4-\tilde{c}_{s}^{0.98}\right) S_{D}\left(\tilde{c}_{s}, 0.49,0.045\right) S_{U}\left(\tilde{c}_{s}, 0.35,0.05\right)
\end{gathered}
$$

$$
\mu_{E}=R T\left(1.36\left(0.74-\tilde{c}_{s}\right)+1.26\right) S_{U}\left(\tilde{c}_{s}, 0.5,0.02\right)
$$

Step up and step down functions respectively are defined by

$$
\begin{gathered}
S_{U}\left(x, x_{c}, \delta\right)=0.5\left(\tanh \left(\frac{x-x_{c}}{\delta}\right)+1\right) \\
S_{D}\left(x, x_{c}, \delta\right)=0.5\left(-\tanh \left(\frac{x-x_{c}}{\delta}\right)+1\right)
\end{gathered}
$$

The stable equilibrium voltage associated with this discharge is plotted in Figure 7. The surface concentration gradient is specified by the non-wetting natural boundary condition, ${ }^{15}$ and the surface flux is imposed by the intercalation current by Equation 4 . Thus, the coupling of the solid model with the remainder of the porous electrode model is the same as the solid solution model.

\section{List of Symbols}

c salt concentration

$c_{\mathrm{s}} \quad$ concentration in the active material

$D \quad$ diffusivity, $\mathrm{m}^{2} / \mathrm{s}$

F Faraday's constant, 96487 C/equiv

$f \quad$ activity coefficient

$G \quad$ Free energy

$i_{n} \quad$ rate of production of positive current in solution, $\mathrm{A} / \mathrm{m}^{2}$

$i_{0} \quad$ exchange current density, $\mathrm{A} / \mathrm{m}^{2}$

$i \quad$ apparent current density $\mathrm{A} / \mathrm{m}^{2}$

$R \quad$ ideal gas constant, $8.314 \mathrm{~J} / \mathrm{mol} \mathrm{K}$

$R_{\text {contact }} \quad$ effective resistance difference to the conducting bulk, $\Omega \cdot \mathrm{m}^{2}$

$R_{\text {film }} \quad$ SEI film resistance, $\Omega \cdot \mathrm{m}^{2}$

$T \quad$ temperature, $\mathrm{K}$

U open-circuit voltage, $\mathrm{V}$

\section{Greek}

$\alpha \quad$ transfer coefficient

$\phi \quad$ potential

$\kappa \quad$ ionic conductivity, $\mathrm{S} / \mathrm{m}$

$\mu \quad$ electrochemical potential

\section{Subscripts}

a anodic

c cathodic

$1 \quad$ electronic phase

2 electrolyte phase

\section{References}

1. M. S. Dresselhaus and G. Dresselhaus, "Intercalation Coumpounds of Graphite," Advances in Physics, 51(1), 1 (2002).

2. X. Y. Song, K. Kinoshita, and T. D. Tran, "Microstructural Characterization of Lithiated Graphite," J. Electrochem. Soc., 143(6), L120 (1996).

3. T. Zheng and J. R. Dahn, "Effect of turbostratic disorder on the staging phase diagram of lithium-intercalated graphitic carbon hosts," Phys. Rev. B, 53, 3061 (1996).

4. K. Persson, V. A. Sethuraman, L. J. Hardwick, Y. Hinuma, Y. S. Meng, A. van der Ven, V. Srinivasan, R. Kostecki, and G. Ceder, "Lithium Diffusion in Graphitic Carbon," J. Phys. Chem. Lett., 1, 1176 (2010).

5. P. Arora, M. Doyle, and R. E. White, "Mathematical Modeling of the Lithium Deposition Overcharge Reaction in Lithium-Ion Batteries Using Carbon-Based Negative Electrodes," J. Electrochem. Soc., 146(10), 3543 (1999).

6. B. Suthar, P. W. C. Northrop, D. Rife, and V. R. Subramanian, "Effect of Porosity, Thickness, and Tortuosity on Capacity Fade of Anode," J. Electrochem. Soc., 162(9), A1708 (2015).

7. M. W. Verbrugge and B. J. Koch, "Electrochemical Analysis of Lithiated Graphite Anodes," J. Electrochem. Soc., 150(3), A374 (2003).

8. M. Doyle, J. Newman, A. S. Gozdz, C. N. Schmutz, and J. M. Tarascon, "Modeling predictions of experimental data from plastic lithium ion cells," J. Electrochem. Soc., 143,1890 (1996).

9. K. E. Thomas, R. M. Darling, and J. Newman, "Modeling of Lithium Batteries," in Advances in Lithium-Ion Batteries, New York, Kluwer Academic Publishers, 2002.

10. T. Fuller, C. M. Doyle, and J. Newman, J. Electrochem. Soc., 141(1), 1 (1994). 
11. R. B. Smith and M. Z. Bazant, submitted 2017. preprint: https://arxiv.org/ abs/1701.08858.

12. T. R. Ferguson and M. Z. Bazant, "Nonequilibrium Thermodynamics of Porous Electrodes," J. Electrochem. Soc., 159, A1967 (2012).

13. Y. Zeng and M. Z. Bazant, SIAM J. Appl. Math., 74, 980 (2014).

14. Y. Li, F. Gabaly, T. Ferguson, R. Smith, N. Bartelt, J. Sugar, K. Fenton, D. Cogswell, A. Kilcoyne, T. Tylisczak, M. Bazant, and W. Chueh, "Transition between particleby-particle and concurrent intercalation in phase-separating battery electrodes," Nat. Mat., 71, 183 (2014).

15. M. Bazant, "Theory of chemical kinetics and charge transfer based on nonequilibrium thermodynamics," Accounts of Chemical Research, 46(5), 1144 (2013).

16. T. R. Ferguson and M. Bazant, "Phase transformation dynamics in porous battery electrodes," Electrochim. Acta, 146, 89 (2014).

17. Y. Guo, R. Smith, Z. Yu, D. E. Efetov, J. Wang, P. Kim, M. Bazant, and L. Brus, "Li intercalation into graphite: direct optical imaging and Cahn-Hilliard reaction dynamics," J. Phys. Chem. Lett., 7, 2151 (2016).

18. A. Acrivos and P. L. Chambre, Ind. Eng. Chem., 49, 1025 (1957).

19. M. J. Matlosz, "Experimental Methods and Software Tools for the Analysis of Electrochemical Systems," Dissertation, University of California, Berkeley, 1985.

20. L. O. Valoen and J. N. Reimers, J. Electrochem. Soc., 152(5), A882 (2005).

21. D. M. Bernardi and J.-Y. Go, "Analysis of pulse and relaxation behavior in lithium-ion batteries," J. Power Sources, 196, 412 (2011).

22. B. Vijayaraghavan, D. R. Ely, Y.-M. Chiang, R. Garcia-Garcia, and R. E. Garcia, "An Analytical Method to Determine Tortuosity in Rechargeable Battery Electrodes," $J$. Electrochem. Soc., 159(5), A548 (2012).

23. http://www.cchem.berkeley.edu/jsngrp/

24. https://peerj.com/articles/cs-54/

25. T. F. Fuller, M. Doyle, and J. Newman, "Relaxation Phenomena in Lithium-IonInsertion Cells," J. Electrochem. Soc., 141(4), 982 (1994).
26. Y. K. Chen-Wiegart, R. DeMike, C. Erdonmez, K. Thornton, S. A. Barnett, and Y. Wang, "Tortuosity characterization of 3D microstructure at nano-scale for energy storage and conversion materials," J. Power Sources, 249, 349 (2014).

27. M. Smith, R. E. Garcia, and Q. C. Horn, "The Effect of Microstructure on the Galvanostatic Discharge of Graphite Anode Electrodes in LiCoO2-Based Rocking-Chair Rechargeable Batteries," J. Electrochem. Soc., 156(11), A896 (2009).

28. A. H. Wiedemann, G. M. Goldin, S. A. Barnett, H. Zhu, and R. J. Kee, "Effects of three-dimensional cathode microstructure on the performance of lithium-ion battery cathodes," Electrochim. Acta, 88, 580 (2013).

29. M. Wissler, "Graphite and carbon powders for electrochemical applications," J. Power Sources, 156, 142 (2006).

30. S. J. Harris, E. K. Rahani, and V. B. Shenoy, "Direct in situ observation and numerical simulations of non-shrinking-core behavior in an MCMB graphite composite electrode," J. Electrochem. Soc., 159(9), A1501 (2012).

31. D. E. Stephenson, E. M. Hartman, J. M. Harb, and D. R. Wheeler, "Modeling of Particle-Particle Interactions in Porous Cathodes for Lithium-Ion Batteries," J. Electrochem. Soc., 154(12), A1146 (2007).

32. R. E. Garcia, Y. M. Chiang, W. C. Carter, P. Limthongkul, and C. M. Bishop, "Microstructural modeling and design of rechargeable lithium-ion batteries," J. Electrochem. Soc., 152(1), A255 (2005).

33. D. R. Baker and M. W. Verbrugge, "Intercalate Diffusion in Multiphase Electrode Materials and Application to Lithiated Graphite," J. Electrochem. Soc., 159(8), A1341 (2012).

34. R. Pollard and J. Newman, J. Electrochem. Soc., 128, 491 (1981).

35. V. A. Sethuraman, N. Van Winkle, D. P. Abraham, A. F. Bower, and P. R. Guduru, "Real-time stress measurements in lithium-ion battery negative-electrodes," J. Power Sources, 206, 334 (2012). 


\section{Erratum: In Situ Observation and Mathematical Modeling of Lithium Distribution within Graphite [J. Electrochem. Soc., 164, E3063 (2017)]}

Karen E. Thomas-Alyea, ${ }^{a}$ Changhoon Jung, ${ }^{b}$ Raymond B. Smith, ${ }^{c}$ and Martin Z. Bazant ${ }^{c}$

${ }^{a}$ Samsung Research America, Burlington, Massachusetts 01803, USA

${ }^{b}$ Samsung Advanced Institute of Technology, Yeongtong-gu, Suwon-si, Gyeonggi-do, Korea

${ }^{c}$ Department of Chemical Engineering, Massachusetts Institute of Technology, Cambridge, Massachusetts 02139, USA

C 2017 The Electrochemical Society. [DOI: 10.1149/2.1201706jes] All rights reserved. Published April 6, 2017.

The list of References should include:

36. T. Ohzuku, Y. Iwakoshi, and K. Sawai, "Formation of Lithium-Graphite Intercalation Compounds in Nonaqueous Electrolytes and Their Application as a Negative Electrode for a Lithium Ion (Shuttlecock) Cell," J. Electrochem. Soc., 140, 2490 (1993). 